\title{
Hegel y las redes lógicas como diseñadoras de la realidad ${ }^{1}$
}

\section{Hegel and logical networks as designer of reality}

\author{
Ricardo Espinoza Lolas ${ }^{2}$, Patricio Landaeta ${ }^{3}$ y Juan Ignacio Arias $^{4}$ \\ Pontificia Universidad Católica de Valparaíso \\ Centro de Estudios Avanzados, Universidad de Playa Ancha
}

Recibido: 15/04/2015

Aceptado: 29/02/2016

\section{Resumen}

Este artículo indaga en el método hegeliano de la Wissenschaft der Logik (WdL), porque este método, ya para la filosofía ya para las ciencias ya para la teoría política, nos permite dar cuenta de lo real tanto de la naturaleza como del hombre. El método

\footnotetext{
${ }^{1}$ Este artículo es parte del Proyecto FIAC N 1108: Fortalecimiento, proyección y efectividad del Doctorado en Filosofía de la Pontificia Universidad Católica de Valparaíso, a partir de su Internacionalización. Y es parte del Proyecto Fondecyt N N 1140973: "Realidad y técnica en Zubiri".

${ }^{2}$ Ricardo Espinoza Lolas es Doctor en Filosofía por la Universidad Autónoma de Madrid y Catedrático de Historia de la Filosofía Contemporánea del Instituto de Filosofía de la Pontificia Universidad Católica de Valparaíso. Visiting Fellow de Goldsmiths, University of London. Es miembro y profesor de la Fundación Xavier Zubiri de Madrid. Es Director del Centro de Estudios Hegelianos y Editor Jefe de la Revista de Estudios Hegelianos (www.revistaestudioshegelianos.cl). Ha ganado tres Proyectos FONDECYT como Investigador Responsable en torno a Zubiri (proyectos con duración de 3 años cada uno): El problema del tiempo en Zubiri (Proyecto $\mathrm{N}^{\mathrm{o}}$ : 1060475), Realidad y cuerpo en Zubiri (Proyecto $\mathrm{N}^{\circ}$ : 1110507) y Realidad y técnica en Zubiri (Proyecto $\mathrm{N}^{\circ}: 1140973$ ). Y ha sido Patrocinador de otros dos proyectos FONDECYT (de Postdoctorado).. Ha escrito y editado seis libros: Realidad y tiempo en Zubiri (Comares, Granada, 2006), Zubiri ante Heidegger (Herder, Barcelona, 2008), Hegel. La transformación de los espacios sociales (Midas, Concón, 2012), Flashback, miradas y gestos (Midas, Concón, 2012), Realidad y ser en Zubiri (Comares, Granada, 2014) y El cuerpo y sus expresiones (Universidad de Granada, Granada, 2014). Ha escrito más de 70 artículos en torno a Deleuze, Zubiri, Heidegger, Hegel, Nietzsche, etc. en revistas indexadas. El tema de su investigación es el análisis de nuevas lógicas que permitan repensar la sociedad civil. E-mail: respinoz@ucv.cl

${ }^{3}$ Doctor en Filosofía por la Pontificia Universidad Católica de Valparaíso y por la Université Paris VIII Vincennes-Saint-Denis, Francia. Actualmente es Investigador del Centro de Estudios Avanzados de la Universidad de Playa Ancha, Chile. Correo electrónico: patricio.landaeta@upla.cl

${ }^{4}$ Doctor en Filosofía, Pontificia Universidad Católica de Valparaíso, Chile. Investigador responsable del Proyecto Fondecyt de Postdoctorado $N^{\circ}$ 3150334: "Aportes para una teoría de la soberanía latinoamericana", patrocinado por el Dr. Patricio Landaeta Mardones, en el Centro de Estudios Avanzados, Universidad de Playa Ancha. Correo electrónico: juanignacioak@gmail.com
} 
es expresión de lo lógico. Y vemos en esto la posibilidad de repensar el presente en sus producciones culturales y naturales como momentos expresivos del diseño lógico. Un diseño que acontece en la misma realidad y que la posibilita como tal. Un diseño que se expresa como un cierto algoritmo constructor de la realidad.

Palabras clave: Ciencia de la lógica, Concepto, Hegel, Lo Lógico, Método

\begin{abstract}
This article explores the Hegelian method Logik der Wissenschaft (WDL), because this method as for philosophy and for science and political theory, allows us to think about the nature and man real. The method is the logical expression. And we see in this the possibility to rethink this in its cultural and natural productions as expressive moments of logical design. A design that happens in the reality and the possible as such. A design that is expressed as a certain algorithm constructor of reality.

Keywords: Concept, Hegel, Method, Science of logic, the Logic.

"El entendimiento determina y mantiene firmes las determinaciones; la razón es negativa y dialéctica, por disolver en nada las determinaciones del entendimiento, es positiva, por engendrar lo universal y subsumir bajo él lo particular"s
\end{abstract}

\title{
Introducción
}

¿Cómo es posible leer a Hegel hoy? ¿ ¿Por qué en Hegel se da un pensamiento que se expresa en redes lógicas? ¿Cómo se articula el método con lo Lógico? ¿Cómo opera su método que comprime múltiple información y que le permite diseñar distintas zonas de la realidad? ¿Por qué Hegel vuelve a ser necesario en tiempos de capitalismo salvaje, modelos de negocio, parámetros de cuantificación científico y de exitismo personal exacerbado? En el fondo nada más que atomismo subjetivo7, como diría Hegel en su época. Tiempos de lobos devorando a otros lobos. ¿Qué es lo más propio cuando todo se ha vuelto instrumento inmediato y abstracto de medición y nos hallamos atrapados en una cierta regla que nos subjetiva y nos indica qué

\footnotetext{
${ }^{5}$ Hegel, G.W.F., "Prólogo de 1812”, en Ciencia de la lógica. Volumen I: La lógica objetiva (1812/1813), Madrid, Abada, 2011, p. 185.

${ }^{6}$ Cfr., Espinoza, R., Hegel y las nuevas lógicas del mundo y del Estado. ¿Cómo se es revolucionario hoy?, Madrid, Akal, 2016.

${ }^{7} \mathrm{La}$ "independencia" de la realidad acontece con la intromisión de la subjetividad en aquel mundo ético, en esta acción aparentemente movida por fines particulares. No en balde en la Enciclopedia al referirse a la sociedad civil hablará del "sistema del atomismo" (Véase. Hegel, G. F. W. Enciclopedia de las ciencias filosóficas, Madrid, Alianza Editorial, 2008, p. 542).
} 
hacer y qué esperar, cuando la ciencia a veces, como dice Heidegger ${ }^{8}$, ya no piensa, no crea, sino simplemente calcula dentro de una regla establecida? En el tiempo "actual" en el que somos y nos movemos al parecer no nos queda "tiempo" para vivir, sino simplemente "sobre-vivir" en la matriz violenta del consumismo inmediato que nos circunda anclados al punto presente de este ahora que se presenta simplemente como todo lo que da sentido al hombre (atrapados al tiempo "vulgar" del reloj, como diría Heidegger en las páginas finales de Sein und Zeit de 1927)"; tiempos de mero "presentismo", lo que Jameson llama "fragmentación" o "presente perpetuo": "Lo que denomino 'fragmentación' se refiere... a este presente perpetuo" 10 . Al parecer, para algunos interesados, que creen en las tesis simplonas de Francis Fukuyama y El fin de la historia y el último hombre de $1992^{11}$, donde el modelo neoliberal ya es la realidad misma en su inmediatez, con su total significación (el horizonte de la presencia "capitalista" que da sentido a todas las cosas), y no hay nada que se pueda esperar o reconciliar porque no hay ni pasado ni futuro sino simple presente eterno, inmediato y abstracto de consumismo salvaje.

Entonces, si el panorama presentado es así, ya no es posible salir de la "época" de este horizonte del tiempo presente en el que somos y vivimos atados, estamos ante el ser en su inmediatez indeterminada, como diría Hegel en la Ciencia de la lógica: "Das Sein ist das unbestimmte Unmitelbare"12. Ante este estado de cosas, se

\footnotetext{
${ }^{8}$ Véase, Heidegger, M., Seminario de Le Thor (1969), Córdoba, Alción Editora, 1995.

${ }^{9}$ Véase, Heidegger, M., “\$81 Die Innerzeitigkeit und die Genesis des vulgären Zeitbegriffes”, en Sein und Zeit, Franckfurt am Main, Vittorio Klostermann, 1977, pp. 555-564.

${ }^{10}$ Jameson, F., Reflexiones sobre la postmodernidad, Madrid, Abada, 2010, p. 55. Véase el excelente apartado "Experiencia temporal", pp. 51-75.

11 "Lo que podríamos estar presenciando no es solamente el fin de la guerra fría, o la culminación de un período específico de la posguerra, sino el fin de la historia como tal: esto es, el punto final de la evolución ideológica de la humanidad y la universalización de la democracia liberal occidental como la forma final de gobierno humano" Fukuyama, F., El fin de la historia y el último hombre, Barcelona, Davinci, 2010, p. 11.

${ }^{12}$ Hegel, G.W.F., Wissenschaft der Logik, Erster Teil, Die Objetive Logik. Ersted Band. Die Lehre vom Sein (1832), Hamburg, Felix Meiner, 1985, p. 68. Usaremos la traducciones del filósofo español Félix Duque publicadas: Hegel, G.W.F., Ciencia de la lógica I. La lógica objetiva, 1 El ser (1812) // 2. La doctrina de la esencia (1813), Madrid, Abada, 2011. Y Hegel, G.W.F., Ciencia de la lógica. Volumen II: La lógica subjetiva. 3. La doctrina del concepto (1816), Madrid, Abada, 2015. Estas traducciones son realmente unas de las mejores traducciones en una lengua distinta a la alemana. El trabajo crítico y de análisis del texto hegeliano es sorprendente por su riqueza, y por los matices mismos que en el castellano ofrece el difícil alemán de Hegel. Y cuando sea necesario trabajar con el texto de la segunda edición póstuma de la WdL usaremos la antigua edición de Rodolfo Mondolfo, en Hegel, G.W.F., Ciencia de la lógica, Buenos Aires, Ediciones Solar, 1982. Pues en esta edición se encuentra la segunda edición de la WdL (1832); texto que todavía no aparece en la actual edición española de la WdL de Abada. Y finalmente usaremos las ediciones alemanas para ambos textos de Hegel de la WdL (1812 y 1832). Estos son las ediciones críticas: Hegel, G.W.F., Wissenschaft der Logik. Ersted Band. Die objetive Logik (1812/1813). Herausgegeben von Friedrich Hogemann und Walter Jaeschke, Hamburg, Felix Meiner, 1978. Hegel, G.W.F., Wissenschaft der Logik. Zweiter Band. Die Subjektive Logik (1816),
} 
necesita otro modo de articulación que exprese complejamente la realidad en y por sí misma, en sus múltiples pliegues, pero que la exprese desde un cierto desarrollo que vaya liberando a todo el sistema de esa esclavitud de las necesidades atadas a la inmediatez del tiempo y del resultado inmediato y abstracto y que se realice una cierta emancipación "de" lo empírico "en" lo empírico mismo ${ }^{13}$.

Esto es lo que se ha propuesto en este artículo mediante nuestro estudio del método hegeliano, como un método liberador del hombre de la inmediatez empírica en la que se mueve naturalmente y lo libera para poder diseñar nuevas formas de producciones culturales. Y este método se expresa en redes lógicas, o dicho simplemente, lo Lógico (das Logische) en sus mediaciones. Se necesita la mediación lógica, esto es, la "formación cultural" (die Bildung), en el pliegue del espíritu, para que el hombre poco a poco pueda elevarse sobre sí mismo, sobre su carácter empírico, animal, de mero presente inmediato al que está sujeto con necesidad ${ }^{14}$. Por eso Hegel, desde la Fenomenología del espíritu (FEN), siempre concibe como fundamental la Bildung, en cuanto es la mediación por excelencia que acontece como formación de la subjetividad a nivel individual, social e histórico ${ }^{15}$, para poder abrir al hombre a algo mejor, por encima de lo inmediato y empírico en lo que está atrapado. ${ }^{16}$

Herausgegeben von Friedrich Hogemann und Walter Jaeschke, Hamburg, Felix Meiner Verlag, 1981. Y Hegel, G.W.F., Wissenschaft der Logik. Erster Teil. Die Objetive Logik. Ersted Band. Die Lehre vom Sein (1832). Herausgegeben von Friedrich Hogemann und Walter Jaeschke, Hamburg, Felix Meiner, 1985.

${ }^{13}$ Muy bien Alberto Toscano señala desde Hegel que el fanatismo se mueve en el elemento de lo abstracto, esto es, de lo inmediato: "Fanaticism, Hegel declared, is enthusiasm for the abstract. The question of abstraction is at the coreof any political and philosophical reckoning with fanaticism. It is abstraction, and the universality or egalitarism that attaches to it, which separates the figurie of the fanatic from that of mere madman, and into the bargain lends fanaticism its allure of extreme danger". Toscano, A., Fanaticism. On the Uses of an Idea, London, Verso, 2010, p. 1.

14 "The inescapable contingences of the natural and social world is one obvious way in the which one's commitments can all fail. Likewise, weakness of will as a failure to acquire a certain kind of skill.. is another. One could obviously extend the list (...) There is, however, yet another way in the which a self-interpreting animal can get it wrong about herself, which has to do with failures internal to the colective form of life itself in which one lives. Hegel's form of sublated Aristotelianism holds that part of the freedom of an action consists in it being 'up to the agent', to do or not do the action...". Pinkard, T., Hegel's Naturalism. Mind, Nature, and the Final Ends of Life, New York, Oxford University Press, 2012 p. 115. Esto acontece solamente en la medida que nos vamos levantando de lo inmediato empírico en lo que nos hemos subjetivado.

${ }^{15}$ Como muy bien dice Pippin: "The entire Phenomenology of Spirit is a meditation on self-consciousness and the coming to self-consciousness of human subjects, especially as a kind of collective subject coming to collective self-consciousness". Pippin, R., Hegel On Self-Consciousness. Dessire and Death in the Phenomenology of Spirit, New Jersey, Princeton University Press, 2011, p. 7.

16 "El comienzo de la formación y del remontarse desde la inmediatez de la vida sustancial tiene que proceder siempre mediante la adquisición de conocimientos de principios y puntos de vista universales, en elevarse trabajosamente hasta el pensamiento de la cosa en general, apoyándola o refutándola por medio de fundamentos, aprehendiendo la rica y concreta plenitud con arreglo a sus determinabilidades, 
Estamos en el asunto mismo del método, que en Hegel se constituye como el sistema de redes lógicas por antonomasia de la realidad en su desarrollo (en su producción) y esto es lo Lógico. Este artículo intentará expresar cómo opera este método a la altura de los tiempos. Para ello es fundamental tener en cuenta que el método no tiene nada que ver con algún rasgo subjetivo de Hegel, ni menos algo así como lo objetivo que permite tener una cierta regla "para" medir la realidad (como si lo "objetivo" fuera algo que está fuera del sujeto y a su vez el "sujeto" fuera del objeto). El método es anterior a la epistemología y ontología sujeto-objeto, porque es la base misma donde ese duplo luego se constituye como tal ${ }^{17}$. El método es expresión sistémica del todo y esto es lo Lógico. Por eso en Hegel este método, con todo lo que ello conlleva, es la filosofía misma en su despliegue y manifestación. Incluso podríamos decir que su filosofía pretende ser "la" filosofía del método: filosofía que funda tanto a las ciencias como a cualquier otra filosofía. El método se convierte en la "llave de bóveda" para ingresar no solamente a la filosofía hegeliana sino a la filosofía misma y, con ello, a todo lo real, ya natural como espiritual. En esto, Hegel se nos actualiza una y otra vez en distintas épocas y nuevos sistemas de pensamientos; y su filosofía nunca envejece y siempre da de sí conceptos que nos permiten ver el estado de la cuestión de lo que acontece a la altura de los tiempos. Es por esto que Hegel se nos vuelve un clásico, tal como sostiene Vincenzo Vitiello: "Porque es un clásico, y los clásicos, se sabe, hablan en el tiempo allende el tiempo: su voz es inextinguible. Esto es verdadero... Porque ningún filósofo ha atravesado el tiempo posterior a su muerte -y dentro de poco serán dos siglos- de modo tan amplio y significativo como Hegel. Ni siquiera Kant: la única figura que se erige a su misma altura." 18

sabiendo bien a qué atenerse y formándose un juicio serio acerca de ella." Hegel, G. W. F., Fenomenología del espíritu, México, FCE, 1966, p. 9.

${ }^{17}$ Como dice claramente el filósofo español Félix Duque en su libro Historia de la Filosofía Moderna. La era de la crítica respecto del método, explicitándolo de manera negativa: "No es una invención u ocurrencia de un individuo llamado Hegel, ni de una comunidad de sabios, ni mucho menos el modo general de proceder en el pensamiento por parte de esa vacua abstracción llamada 'hombre', tan mentada por algunos filósofos. En suma, el método no es algo 'subjetivo' (en el sentido de: aplicado a... y organizador de algo que está 'ahí fuera' y es tildado de 'objetivo'). Tampoco es un recetario de 'instrucciones de uso' para 'mejorar' o enmendar nuestra inteligencia o nuestro 'espíritu', sean éstos lo que fuere. No es una 'fundamentación' de las ciencias ni de la praxis, en el muy amplio sentido de ambos términos... mejores ciudadanos ni cristianos (o miembros de cualquier confesión); ni siquiera nos hace mejores 'personas'. En suma: el método no es edificante” Duque, F., Historia de la Filosofia Moderna. La era de la crítica, Madrid, Akal, 1998, pp. 600-601.

${ }^{18}$ Vitiello, V., "Por qué Hegel, Hoy", en Espinoza, R. (ed.), Hegel. La transformación de los espacios sociales, Concón, Midas, 2012, p. 17. 
¿Qué pasa más precisamente con el método hegeliano de la Wissenschaft der Logik? Es necesario decir desde el comienzo, para evitar confusiones, que no puede haber un método dialéctico (lo dialéctico es un momento del método, pero no "el" método mismo ${ }^{19}$ ): el método solamente es método y nada más que método, absolutamente método, esto es, método simpliciter, por antonomasia, de suyo. El método no tiene adjetivo, no es dialéctico ni nada parecido, es simplemente método. El método es método absoluto y lo absoluto mienta ab-suelto, esto es, método en libertad o, mejor dicho, método que libera a lo real de lo inmediato empírico (ya la naturaleza como el hombre), lo libera de su necesidad, de su carácter sustancial. Hegel en "La doctrina del concepto” de 1816, lo señala así: “... der Begriff die Wahrheit der Substanz, und indem die bestummte Verhältnissweise der Substanz, und die Nothwendigkeit, und als die Verhältnissweise des Begriffs" ${ }^{20}$. El método es expresión misma de la libertad en los pliegues de lo real. Método y libertad son sinónimos para Hegel. Método es la expresión misma del acontecer de la mediatización de lo inmediato $^{21}$ : la libertad. En Hegel nunca la libertad se entiende desde la concepción del "libertus", el estado que cobraba el "servus" cuando se le daba la libertad en Roma. O sea, el libertus era el que abandonaba las "cadenas" de la necesidad. Nada más distinto en Hegel. Esa definición sería meramente inmediata y empírica. La libertad va de la mano con la negatividad en su proceso metódico. La libertad indica ese levantamiento en desarrollo de lo empírico sobre sí mismo. De lo inmediato en su propia mediación. Es lo inmediato mismo que se va abriendo y en ello desbrozando de su atadura inmediata empírica. Y en esto lo inmediato se eleva. Por eso, método

\footnotetext{
19 “[...] el método no admite más calificativo que el de 'absoluto'. O sea: no hay un 'método dialéctico"” Duque, F., "La era...", op. cit., p. 601. Esta comprensión del método contrasta con la vasta interpretación heideggeriana. Véanse, por ejemplo, los siguientes libros: Heidegger, M., Identidad y diferencia, Barcelona, Anthropos, 1988; Caminos del bosque, Madrid, Alianza, 2010; Hegel, Buenos Aires, Prometeo, 2005; Fink, E., Hegel: Interpretaciones fenomenológicas de la Fenomenología del espiritu, Barcelona, Herder, 2011; Zubiri, X., Naturaleza, Historia, Dios, Madrid, Alianza, 2007; Sobre la esencia, Madrid, Alianza, 2008; Los problemas fundamentales de Occidente, Madrid, Alianza, 1994; etc. En la Escuela fenomenológica de Freiburg se lee a Hegel desde el sujeto, la razón, el Estado, la totalidad, esto es, desde la dialéctica. Y desde este modo interpretativo, el hombre queda olvidado en cuanto a su carácter existencial y único. Por ejemplo, Heidegger señala esto a lo largo de muchos años y esta interpretación perdura y no cambia. Esta cita es de 1958: "La dialéctica es el proceso de la producción de la subjetividad del sujeto absoluto y, en cuanto tal, es su 'actuación necesaria"'. Heidegger, M., "Hegel y los griegos", en Hitos, Madrid, Alianza, 2001, p. 348.

${ }^{20}$ Hegel, G.W., Wissenschaft der Logik. Zweiter Band. Die Subjektive Logik (1816), op. cit., p. 12. “... el concepto es la verdad de la sustancia; y como el modo determinado de relación propio de la sustancia es la necesidad, la libertad se muestra como la verdad de la necesidad y como el modo de relación del concepto" Hegel, G.W.F., Ciencia de la lógica. Volumen II: La lógica subjetiva. 3. La doctrina del concepto (1816), op. cit., p. 126.

${ }^{21}$ Cfr. Hegel, G.W.F., en "Enciclopedia...”, op. cit., §23, p. 130.
} 
no solamente va con libertad, sino obviamente con "formación cultural" (Bildung), tal como citamos arriba.

Hablar del método en filosofía es sumergirnos tanto en lo epistemológico como en lo ontológico que da cuenta de cómo poder entender la realidad en todas sus manifestaciones tanto racionales como reales (usando la terminología de Hegel que ha quedado como un sintagma en el Prólogo de su Filosofía del derecho) ${ }^{22}$. El método nos lleva al problema del camino (que es lo que indica el término en su raíz etimológica griega, esto es, andar de camino, en camino), un camino que a veces supone relatos, esto es, los puntos tanto del inicio como del fin del camino propiamente tal (este es el modo simple de entender el método). Pero otras veces el método indica un camino en el caminar mismo donde los relatos son momentos dinámicos de ese caminar, son "puestos" por el caminar en tanto caminar ${ }^{23}$; de esta manera es desde donde Hegel estará pensando el camino por excelencia de la libertad: de la liberación de lo empírico, de lo natural, de lo inmediato, de su desbrozamiento pero en lo empírico mismo, en esa misma inmediatez que se nos aparece y brilla como "la" naturaleza (tan cara a Hölderlin y Schelling) ${ }^{24}$. El camino de todos los caminos, el camino que

22 “... Lo que es racional es real y lo que es real es racional”. Hegel, G.W.F., Rasgos Fundamentales de la Filosofía del Derecho, Madrid, Biblioteca Nueva, 2000, p. 74. La interpretación que hace Žižek de este texto se ajusta a nuestra comprensión, pues señala justamente aquello que queremos expresar mediante la idea del método, el lugar que ocupa lo otro que queda fuera y que no es sistematizable, la contingencia, en el pensamiento de Hegel: “'Lo real es racional' (y viceversa) no debe leerse de ningún modo como 'todo lo que es real es racional', sino como 'no hay nada de lo real que no sea racional'; el precio que hay que pagar por ese 'sin excepción' es justamente que no todo es racional, que existe el dominio de la contingencia que escapa a la deducción conceptual...”. Žižek, S., El más sublima de los histéricos, Barcelona, Paidós, 2013, p. 40.

${ }^{23}$ Más exactamente, cuando lo buscado en el camino se identifica con el mismo camino: el método unifica camino y meta, instrumento y objeto, de ahí, como se señaló su etimología en la voz griega, donde methodos se compone de las palabras meta y hodós, la meta, el más allá, se encuentra en el camino mismo y por este camino. De ahí la identificación de Hegel entre lo conocido y el método. Pues si el método fuera algo distinto al conocimiento, nunca podríamos conocer la cosa, sino que sólo nos quedaríamos amparados en el mero gesto de tender hacia ella. "si el examen del conocimiento que nos representamos como un médium nos enseña a conocer la ley de su refracción, de nada servirá que descontemos ésta del resultado, pues el conocimiento no es la refracción del rayo, sino el rayo mismo a través del cual llega a nosotros la verdad y, descontado esto, no se habría hecho otra cosa que indicarnos la dirección pura o el lugar vacío." Hegel, G. W. F., "Fenomenología...”, op. cit., p. 52. Pippin lo indica de este modo: "Some of the most influencial and powerfull claims, in The Philosophie of Rigth, it would certainly seem, can be economically disscused as matters of political theorie, alone". Pippin, R., Hegel's Practical Philosophy. Rational Agency as Ethical Life, Cambridge, Cambridge University Press, 2010, p. 123.

24 "En tanto la filosofía de la naturaleza es consideración concipiente, tiene por objeto el mismo universal, pero para sí, y lo contempla en su propia e inmanente necesidad con arreglo a la autodeterminación del concepto". Hegel, G.W.F., "Enciclopedia...", op. cit., p. 304. Véase el excelente estudio de José María Ripalda: Ripalda, J.M., "Introducción”, en Hegel, G.W.F., Filosofía Real, Madrid, FCE, 2006, pp. xii - liii. En este estudio se muestra en detalle el viraje dificultoso del propio Hegel. Todo su intento para salir del horizonte de Hölderlin y Schelling y levantar su propia filosofía. 
permite dar a la vez con ambos momentos del camino, por una parte, con el hombre y, por otra parte, con la naturaleza. Dicho con el filósofo Ramón Valls Plana, para lo medular del pensamiento hegeliano que ya se expresa en FEN: "La Fenomenología sigue el movimiento de la existencia, lo observa y registra sin intervenir en él, pero al mismo tiempo lo interpreta, eso es, explicita la correspondencia de este movimiento con el movimiento esencial. Y no se olvide que ambos aspectos son inseparables. Al hacer la descripción nos avisa Hegel que operamos una abstracción de la contingencia de la persona y sus motivaciones particulares. ¿Y dónde estaría la clave para esta abstracción si no se posee ya el saber absoluto que permite distinguir lo contingente de lo necesario? Toda narración abstrae o selecciona, toda historia se escribe desde una metafísica más o menos confesada" ${ }^{25}$. En el método como plasmación de lo Lógico se da la articulación, el nexo mismo desde dónde es posible hablar ya de hombre ya de la naturaleza; lo Lógico es lo que permite el estructurar lo que sea ${ }^{26}$.

Luego el método no siempre se entiende como un puente entre dos momentos sino lo que permite en sí mismo constituir esos momentos e incluso el puente mis$\mathrm{mo}^{27}$. El método es la matriz absuelta misma que expresa en complejidad toda la realidad en sus distintos pliegues racionales como reales.

\section{II}

El método expresa en complejidad toda la realidad, acabamos de sostener, siendo, a la vez, la misma expresión del método algo complejo ${ }^{28}$. El método lógico,

\footnotetext{
${ }^{25}$ Valls Plana, R., Del yo al nosotros. Lectura de la Fenomenología del Espiritu de Hegel, Barcelona, Editorial Estela, 1971, p. 47.

26 "Las formas del pensamiento están ante todo expuestas y consignadas en el 'lenguaje' del hombre. En nuestros días nunca se repetirá bastante que el hombre sólo se distingue de los animales por el pensamiento. En todo aquello que se le convierte en algo interior, y principalmente en la representación, en lo que hace suyo, ha penetrado el lenguaje; y lo que el hombre convierte en lenguaje y expresa con él, contiene escondida, mezclada o elaborada una categoría; tan natural es al hombre el elemento lógico, o para decirlo mejor, tan propio es de su 'naturaleza' misma". Hegel, G.W.F., Ciencia de la lógica, 1982, op. cit., p. 18.

${ }^{27}$ En efecto, si el puente fuese otro a los momentos y no parte esencial de ellos, la unión sería meramente extrínseca, una mera astucia del conocimiento. Recuérdese en esto la conocida metáfora fenomenológica: "si el instrumento se limitara a acercar a nosotros lo absoluto como la vara con pegamento nos acerca el pájaro apresado, sin hacerlo cambiar en lo más mínimo, lo absoluto se burlarla de esta astucia, si es que ya en sí y para sí no estuviera y quisiera estar en nosotros; pues el conocimiento seria, en este caso, en efecto, una astucia, ya que con sus múltiples afanes aparentada algo completamente diferente del simple producir la relación inmediata y, por tanto, carente de esfuerzo." Hegel, G. W. F., "Fenomenología...", op.cit., pp. 51-52.

${ }^{28}$ Deleuze en su tesis doctoral de 1968: Spinoza y el problema de la expresión señala algo muy interesante sobre el carácter mismo del ser expresivo del pensamiento de Spinoza, carácter dual que nos permitirá ver a contra luz al método hegeliano, es por ello que citaremos in extenso el texto de Deleuze, pues en él podemos ver cómo opera y se da la complejidad misma del método que nos permite describir
} 
dado su carácter expresivo y dual, es tanto desarrollar como englobar o, si queremos, tanto ex como in; por el primero se exterioriza: espacio y, por el segundo, se interioriza: tiempo; esto es, se sale fuera de sí y es naturaleza; y se reflexiona sobre sí y es espíritu; aquí ya podemos ver el carácter complejo del método y por eso su riqueza a la hora del análisis de la realidad. O como lo dice Vitiello: “... la Lógi$c a$ es esencialmente ex-posición, construcción ex-plicativa del yo y del mundo, del mundo y del yo, a partir de lo profundo" 29 . El método como lo lógico siempre se despliega y en lo desplegado mismo, por una parte, se engloba, se reflexiona, se espiritualiza y, por otra parte, se desarrolla, se exterioriza, se naturaliza. El método es en sí mismo expresión que desarrolla y engloba, se extiende y se interioriza, de allí, como dijimos, su complejidad en la simplicidad misma de su expresión. ¿Expresión desarrollante y englobante de qué? De nada distinto del método mismo. El método como estructuración de lo Lógico es método y nada más que método, pero en esta aparente tautología vacía, inmediata y natural, se da la expresión y acontece así la mediación misma: lo libre (mediación fundamental en el pensamiento de Hegel en general y en la WdL en especial). Tal mediación en la que consiste el método como matriz lógica mienta por lo menos dos perspectivas de análisis. Una epistemológica centrada en el hombre y otra centrada en las cosas, que es la ontológica. Por tanto, el método expresa la realidad tanto como exterior y como interior, ya desde una mirada epistemológica ya ontológica. Cada una de estas batientes son caras de lo mismo en su expresión metódica, esto es, de lo Lógico (das Logische). Es todo el método que expresa lo ontológico y es todo el método que expresa lo epistemológico. Lo Lógico en una primera determinación es la matriz misma metódica absuelta y sistémica que se expresa a sí misma en tanto lo epistemológico como lo ontológico ${ }^{30}$.

\footnotetext{
la realidad: "La palabra 'expresar' tiene sinónimos. Los textos holandeses del Tratado breve emplean uytdrukken-uytbeelden (a la vez manifestar y demostrar): la cosa pensante se expresa en una infinidad de ideas correspondientes a una infinidad de objetos; pero igualmente la idea de un cuerpo manifiesta a Dios inmediatamente; y los atributos se manifiestan ellos mismos por ellos mismos. En el Tratado de la reforma, los atributos manifiestan la esencia de dios: ostendere. Pero no son los sinónimos lo más importante. Más importante son los correlativos, que precisan, acompañan la idea de expresión. Esos correlativos son explicare e involvere. Así, la expresión no solamente se dice que expresa la naturaleza de la cosa definida, sino que la engloba y la explica. Los atributos no sólo expresan la esencia de la substancia; ora la explican, ora la engloban. Los modos engloban el concepto de dios al mismo tiempo de expresarlo, de manera que las ideas correspondientes engloban ellas mismas la esencia eterna de Dios (...) Explicar es desarrollar. Englobar es implicar. Sin embargo, ambos términos no son contrarios: sólo indican dos aspectos de la expresión” Deleuze, G., Spinoza y el problema de la expresión, Barcelona, Atajos, 1999, pp. 11-12.

${ }^{29}$ Vitiello, V., op. cit., p. 185.

${ }^{30}$ Para un estudio detallado de la estructura de la WdL, véase, de Duque, F., "Estudio preliminar", en Hegel, G.W.F., Ciencia de la lógica, 2011, op. cit., pp. 13-138.
} 
Si nos damos cuenta en el análisis anteriormente hecho, Hegel está realizando una transformación no solamente en lo filosófico sino en el modo mismo de hacer filosofía y de enfrentar los problemas. Hegel en la era analógica, de su época del siglo XIX, ya estaba diseñando la matriz lógica de lo digital que vendría con todo en la segunda mitad del siglo $\mathrm{XX}^{31}$. Miremos esto con algún detalle. Hegel crea una matriz lógica que le permite dar cuenta de los pliegues de la realidad en su manifestarse inmediato, en su ser, y en distintas épocas históricas, en su esencia. Esto es, estaba digitalizando y con ello comprimiendo toda la realidad a elementos lógicos y tales elementos constituyen dos pliegues de consideración. Lo Lógico es, si se quiere actualizar la terminología, el algoritmo que le permite a Hegel describir en digital toda la información de un momento en la inmediatez, "aparentemente dada", de la realidad (lo que Hegel llama: ser, Sein, en la WdL) y en ello, a la vez, ver cómo se dio, aconteció, tal momento históricamente en la tensión dialéctica propiamente tal (lo que se llama: esencia, Wesen, en la WdL) y finalmente poder desarrollar en lo digital mismo de su algoritmo lógico cómo se puede seguir diseñando y produciendo efectivamente en la realidad (lo que se llama: concepto, Begriff, en la WdL). Hegel lo dice así en "La doctrina del concepto" de la WdL: "Die objective Logik, welche das Seyns und Wesen betrachtet, macht daher eingentlich die genetische Esposition des Begriffes aus. Näher ist die Substanz schon das reale Wesen, oder das Wesen, in so fern es mit dem Seyn vereignit und in Wirklichkeit getreten ist. Der Begriff hat daher die Substanz zu seiner unmittelbaren Voraussetzung, sie ist das an sich, was er als manifestirtes ist" 32 . Esto es desde un primer momento en que "así" son las cosas (tal como se presentan en el ser) a un segundo momento de un porqué llegaron a ser así las cosas, a través de toda la carga dialéctica (el momento de fundamentación esencial),

\footnotetext{
31 Véanse los siguientes artículos de: Castells, M., "Creatividad, innovación y cultura digital. Un mapa de sus interacciones", en Telos, cuadernos de comunicación e innovación, 77, 2008, pp. 50-52. Por ejemplo, en dicho artículo en la red dice: “... el proceso de desarrollo del conocimiento en nuestros tiempos o, lo que es lo mismo, el proceso de creatividad y su derivada, la innovación, flotan en nuestro entorno a través de su modo de comunicación y en la tecnología de éstas, basadas en la microelectrónica digitalizada, transmitida y construida a través de las telecomunicaciones, redes informáticas y bases de datos". También el artículo: "La apropiación de las tecnologías: cultura juvenil en la era digital", en Telos, cuadernos de comunicación e innovación, 81, 2009, pp. 111-113. "La democràcia en l'era d'Internet", en Via valors, Idees, Actituds: Revista del Centre d'estudis Jordi Pujol, 12, 2010, pp. 7-13.

${ }^{32}$ Hegel, G.W.F., Wissenschaft der Logik. Erster Band. Die Objektive Logik (1812/1813), op. cit., p. 11: "La lógica objetiva, que toma en consideración el ser y la esencia, constituye propiamente, en consecuencia, la exposición genética del concepto. Vista más de cerca, la sustancia es ya la esencia real, o sea la esencia en cuanto que está unificada con el ser y ha entrado en la realidad efectiva. El concepto tiene por consiguiente a la sustancia como presuposición inmediata suya: ella es en sí lo que él es como lo manifiesto" Hegel, G.W.F., Ciencia de la lógica. Volumen II: La lógica subjetiva. 3. La doctrina del concepto (1816), op. cit., p. 125.
} 
a un momento en que las cosas se siguen diseñando, sin parar (de modo histórico), una y otra vez (el momento del concepto en su realización efectiva).

Pero vayamos por partes, el método es lo Lógico, y lo Lógico es expresión de la realidad en todos sus pliegues. Por eso lo uno siempre acontece inmediatamente como múltiple para Hegel desde la certeza sensible (FEN) o desde el ser (WdL). Esto lo expresa muy bien Vitiello: "El inicio de la Lógica es la negación del Inicio. Lo Uno desde siempre es múltiple." 33 Como expresión el método funciona como una matriz de diseño que a su vez expresa lo que aparece en su inmediatez (el momento de ser en su multiplicidad necesaria) como, por otro lado, muestra cómo se agencia cualquier tipo de producción que aparece en la tensión dialéctica del movimiento mismo de múltiples momentos de unos contra otros en la historia (el momento de esencia) y que finalmente esté siempre operando en diseño real, creador y efectivo de todos esos pliegues (el momento de concepto).

Si lo queremos precisar, ese carácter de expresión del método lógico complejo es el diseño mismo que da de sí todo: "el" algoritmo de todos los algoritmos, diseño que explicita y engloba. Esa expresión la podemos reunir por simplicidad en los dos planos antes señalados, como son el epistemológico y el ontológico. Con plano epistemológico nos referimos al hombre, las producciones culturales y con plano ontológico a las cosas, la naturaleza. El método en su expresión compleja mienta esa doble bisagra de lo epistemológico-ontológico, en la que Hegel es maestro de este doble momento, tal como queda manifiesto desde su primera obra publicada. Hay pasajes en FEN que se escriben y redactan pensados desde este doble momento de análisis. El filósofo alemán no le dice al lector en qué plano está, da por hecho que éste ha asumido que lo Lógico es metódico y que el método en su simplicidad es expresividad de complejidad y que la expresión es de los múltiples pliegues de la realidad en su doble momento epistemológico-ontológico ${ }^{34}$. En esta obra Hegel siempre escribe de este modo (por ejemplo, en el capítulo tercero de las fuerzas y el entendimiento esto es muy evidente) $)^{35}$, de allí lo fascinante de la aventura de leerla

\footnotetext{
${ }^{33}$ Vitiello, V., op. cit., p. 183.

${ }^{34}$ Tal como lo señala en el Prólogo de esta obra: "Tal vez podría considerarse necesario decir de antemano algo más acerca de los diversos aspectos del método de este movimiento o de la ciencia. Pero su concepto va ya implícito en lo que hemos dicho y su exposición corresponde propiamente a la Lógica o es más bien la Lógica misma. El método no es, en efecto, sino la estructura del todo, presentada en su esencialidad pura." Hegel, G. W. F., "Fenomenología...", op. cit, p. 32.

${ }^{35}$ Véase el capítulo de FEN "Fuerza y entendimiento". Ya el título de este capítulo mienta las dos caras del análisis de Hegel. Se podría decir algo así: lo que en la esfera de lo real es la Fuerza y es la esfera del hombre el Entendimiento. Ahí se ven los dos pliegues de análisis: "El concepto de fuerza se mantiene más bien como la esencia en su realidad misma; la fuerza como real sólo es pura y simplemente en la exteriorización, que no es, al mismo tiempo, otra cosa que un superarse a sí misma. Esta fuerza real, representada como libre de su exteriorización y como algo que es para sí, es la fuerza repelida hacía sí misma; pero esta determinabilidad es, de hecho, a su vez, como ha resultado, sólo un momento de la exteriorización. [...] La realización [Realisierung] de la fuerza es, por consiguiente, al mismo
} 
una y otra vez, y donde radica, a la vez, la gran dificultad para comprenderla. Su carácter tan enigmático como seductor se encuentra, en parte, en ello. Sin embargo, en la WdL ("La doctrina del ser" de 1812, "La doctrina de la esencia" de 1813, "La doctrina del concepto" de 1816 y segunda edición póstuma de "La doctrina del ser" de 1832) nos muestra ya de forma acabada y pulida la matriz misma lógica que antes no le permitió escribir FEN de forma adecuada y, de allí, el posterior rechazo de Hegel a este libro. Más adelante, en las lecciones que Hegel realizó en Berlín, acontece la claridad de un filósofo maduro y sereno con experiencia y que quiere ser entendido. Hegel siempre estaba escribiendo, dando clases y pensando en uno de los momentos de la expresión metódica, el momento que va a las producciones humanas y naturales en su desarrollo histórico. ${ }^{36}$

Otro momento de este carácter expresivo del método en su absolutez es el carácter dinámico del mismo. El método no opera en nada sincrónico sino que todo está siempre "en proceso", "en devenir", movimiento o, si se quiere, dicho de modo más simple y tosca, "en historia". El método es formalmente dinámico. El método es de suyo dinámico y tal expresión dinámica mienta que los pliegues expresivos epistemológicos como ontológicos están en devenir, en proceso y no pueden no estarlo porque lo Lógico acontece dinámicamente en la inmediatez misma de lo humano como de la naturaleza. Este es el motivo de por qué no hay fin posible de la historia en Hegel. ¿Qué sería un fin de la historia ya al modo trascendente como utópico o político o económico, etc.? No es posible el corte final al interior de la filosofía de Hegel (en esto es completamente distinto a Hölderlin, Schelling ${ }^{37}$ o, posteriormen-

tiempo, la pérdida de la realidad [Realität]; la fuerza ha devenido así más bien algo totalmente otro, a saber: esta universalidad que el entendimiento conoce primeramente o de un modo inmediato como su esencia y que también como su esencia se muestra en su realidad [Realität] que debiera ser, en las sustancias reales. [...]Si consideramos el primer universal como el concepto del entendimiento, en el que la fuerza no es todavía para sí, el segundo será ahora su esencia, tal y como se presenta en y para sí. O, a la inversa, si consideramos el primer universal como lo inmediato, que debiera ser un objeto real para la conciencia, tenemos que el segundo se halla determinado como lo negativo de la fuerza sensible objetiva; es la fuerza tal y como es en su verdadera esencia, solamente en cuanto objeto del entendimiento; aquel primero sería la fuerza repelida hacía sí misma o la fuerza como sustancia; este segundo, en cambio, lo interior de las cosas como interior, que es lo mismo que el concepto como concepto.". Hegel, G. W. F., "Fenomenología...", op. cit, p. 88. Véase, además, el excelente texto: Ripalda, J.M., "Fuerza y Entendimiento. El conflicto de la especulación y la realidad", en Duque, F. (editor), Hegel: La Odisea del Espíritu (1807-2007), Madrid, Círculo de Bellas Artes, 2010, pp. 69-83. ${ }^{36}$ También se ha de destacar que esto se debe a que el carácter lógico de FEN se debe al valor que tiene en esta obra la proposición especulativa. No es sino hasta los años 1810-1811, cuando Hegel descubre el "valor especulativo del silogismo", momento en que la Lógica asumirá la forma de exposición como se conocerá desde el años siguiente. Cfr. Duque, F., Estudio preliminar, en Hegel, G.W.F., Ciencia de la lógica I., 2012, op. cit.

${ }^{37}$ Lo que Hegel está mostrando en este primer nivel de análisis del método, el nivel del ser en su inmediatez, es, por decirlo de modo simple, llevar a precisión "conceptual" tanto el "cantar poetizante" de su amigo Hölderlin, como también esa "filosofía estetizante", no del todo fraguada, de su otro amigo 
te, Heidegger), así como tampoco es posible la "cosa en sí". De allí sus estudios de arte, religión, ciencia, etc., de un modo histórico, siempre en proceso y siempre intentando liberar lo empírico mismo de sus estofas materiales, de su naturaleza; la materialidad misma se va transparentando, clarificando, se va "digitalizando", siempre está en la muerte de lo analógico (una cierta muerte no solamente del arte, sino también de la religión, de la propia filosofía, etc.). Se piensa todo, incluso el mismo pensamiento, desde ese carácter histórico, tenso, "liberador". Cada uno de estos vectores, de clúster de cosas, son vistos de un modo dinámico y operativo: arte, religión, política, ciencia, etc. Y además cada uno de estos despliegues de producciones sociales y naturales no están en compartimentos estancos, sino articulados los unos con los otros: lo religioso, lo estético, lo político, lo científico, a la vez, están estructurados en la matriz lógica: de allí su carácter sistémico. Cada uno de estos operadores y muchos otros que van naciendo a lo largo de la historia: arte, religión, filosofía, etc., están entrelazados los unos con los otros y, por ello, cada uno de estos operadores dinámicos se entrecruzan en esos nudos lógicos que son sus conectores y que articulan el diseño mismo que expresa y despliega la realidad. Así es la historia de la filosofía, es la historia de todas las producciones y, a su vez, lo histórico mismo en expresión de esas líneas nodales de articulación de todo que se presenta en la inmediatez empírica de su ser ${ }^{38}$.

\section{III}

Finalmente, asumiendo lo hasta acá dicho, pensemos los momentos del método tal como lo propone Hegel en su Enciclopedia: "a) El pensamiento en cuanto entendimiento se queda parado en la determinidad fija y en la distintividad de ella frente a otra; un tal abstracto [así] delimitado vale para el entendimiento como siendo de suyo y como subsistente. b) El momento dialéctico es el propio superar de tales determinaciones finitas y su pasar a sus opuestas... c) Lo especulativo o racional-positivo aprehende la unidad de las determinaciones en su oposición, lo afirmativo que

Schelling. Se tiene que dar densidad conceptual a esa Belleza (Schönheit), que acontece en el poetizar de Hölderlin, como a esa Naturaleza (Natur) en el filosofar de Schelling. Cfr. Espinoza, R., "Arte y religión en la Phänomenologie des Geistes de Hegel a la luz de la Wissenschaft der Logik...", en Daimon, 43, 2008, pp. 71-91. Aquí se explica en detalle esta articulación entre estos autores.

${ }^{38}$ Cfr. Espinoza, R., "Una lectura 'desmesurada' de la Ciencia de la lógica de Hegel: en torno a 'la línea nodal de relaciones de medida", en Philosophica, 30, 2007, pp. 89-102. "Este artículo mienta lo medular del Libro de "La doctrina del ser", esto es, el problema del ser a la luz de cierta medida que regla y regular la totalidad en su manifestación presente. Y en esto encontramos puntos de concordancia con la Escuela fenomenológica en general y con Zubiri en especial. Por ejemplo, véase, la Trilogía de la Inteligencia sentiente. Zubiri, X., Inteligencia sentiente, Inteligencia y Logos, Inteligencia y Razón (todos publicados en Alianza, Madrid). En estos tres libros se puede apreciar como lo Lógico es fundamental en la construcción de los contenidos de lo real y además a modo histórico. 
se contiene en la disolución de ellas y en su pasar" ${ }^{39}$. En un lenguaje epistemológico, el método se muestra desde tres rasgos bien característicos del pensamiento del Idealismo alemán: el entendimiento, la razón negativa y la razón afirmativa. Es, si se quiere, el ámbito de la representación, el carácter dialéctico negativo y la afirmación de lo que emerge como síntesis que se despliega y produce. Dicho método en un plano ontológico es más simple, esto es, ser (Sein), esencia (Wesen) y concepto (Begriff). Tal división es, como se sabe, la división de los tres libros de la WdL. El ser es al entendimiento como la esencia es a la razón negativa y, finalmente, como el concepto es a la razón afirmativa (que no es nada distinto a la razón negativa como al entendimiento, es la unidad que los integra afirmativamente, esto es, si se quiere, el espíritu). El ser se mueve en el horizonte del teatro de la representación, la que opera en su inmediatez indeterminada o sea, como sistema referencial, de relaciones de medida, de creencias, de dispositivos, de ideologías, de naturalizaciones, de ¡amén y así sea! Por este motivo el libro "La doctrina del ser" de la WdL termina con el gran acápite de la "medida", pues en la "medida" se juega la verdad del ser como lo inmediato indeterminado, y es a su vez el nexo a "El libro de la esencia". Tal inmediatez está mediatizada, es en el fondo un aparecer de las relaciones de medida; y aquí al final de este acápite ya estamos en los dominio de la esencia, esto es, la historia. El ser, en el fondo, es la relación de medida de lo Lógico por excelencia que nos permite medir y ser medidos, el que aparece, en un primer momento no reflexivo, ni mediatizado, como una inmediatez que es así y no de otra manera. En este carácter de relación de medida de lo Lógico crea, a través de la historia (la esencia) y sus producciones culturales, el sistema de referencias y valores que nos permita leer y deletrear la realidad, de esta manera, organizarnos y constituirnos (y esto se nos vuelve en nuestro más brutal panóptico).

La relación de medida por excelencia en la que el ser consiste en su presencia inmediata, y alumbra y brilla es la regla que los griegos se dieron para organizarse como pueblo en ese mundo concreto: la polis. He aquí el pleno significado del "concepto" para los griegos. Y la polis mienta el ser ciudadanos de una forma determinada, eso fue Grecia en su atisbar el ser como presencia: inmediatez indeterminada (naturaleza, belleza; Schelling y Hölderlin de la mano), esto es, relación de medida (regla), esto es, la polis.

El libro "La doctrina de la esencia" es la mediación misma del ser (de lo in-mediato), es su interiorización, su flexión, su vuelta sobre sí; su densificación en capas y capas de pasado. Hegel siempre es muy claro en su decir: "Die Wahrheit des Seyns ist das Wesen" ${ }^{40}$. La esencia es todo el dinamismo mismo, esto es, la historia en su historizar dinámico: "La esencia es el ser asumido. Es simple igualdad consigo

\footnotetext{
${ }^{39}$ Hegel, G.W.F., "Enciclopedia...”, op cit., pp. 183-184.

${ }^{40}$ Hegel, G.W.F, "Die Lehre vom Wesen", en Wissenschaft der Logik. Ersted Band. Die objektive Logik (1812-1813), Felix Meiner Verlag, Hamburg, 1978, p. 241.
} 
misma pero, en esa medida, es la n e g a c i ó n de la esfera del ser en general." ${ }^{{ }^{41}}$ Lo inmediato asumido es la esencia. Al estar asumido como tal, deja en el preciso momento de ser inmediato, es un inmediato "mediatizado". Tal inmediato mediatizado (in-mediato), es la flexión sobre sí mismo, esto es, "reflexión". Por eso "La doctrina de la esencia" puede ser considerado como el libro de la reflexión. Lo propio de la esencia es ser reflexión, el ser se interioriza, podríamos decir que la superficie del ser se va plenificando, aparece todo lo que ha sido su pasado que permite tal superficie (la palabra para "esencia" en alemán "Wesen" literalmente significa lo "sido" y de ahí la cercanía de Hegel con Aristóteles), por esto se vuelve sobre sí, se "flecta" y en ello acontece, como tal, la esencia, la cual estaba oculta y casi olvidada detrás del brillo inmediato del ser que nos subjetiva. La naturaleza se espiritualiza y muestra ciertas regularidades que estaban en el fondo, las que acontecían en los fenómenos como tales, llevando dentro de sí lo que siempre se dejó fuera: el dolor, el residuo, lo que sobra, los que sobran, esto es, la negatividad: su dialéctica ${ }^{42}$ : "En cuanto que el saber quiere conocer lo verdadero, lo que el ser es en y para sí, no se está quieto cabe lo inmediato y sus determinaciones, sino que lo atraviesa de parte a parte, con la presuposición de que d e $\mathrm{t} \mathrm{r}$ á $\mathrm{s}$ de este ser hay aún algo otro que el ser mismo, $\mathrm{y}$ de que este tras-fondo constituye la verdad del ser. Este conocimiento es un saber mediato, pues no se encuentra inmediatamente cabe la esencia ni dentro de ella, sino que comienza a partir de un otro, del ser, y tiene que hacer un camino previo, el camino de sobrepasar el ser o más bien de intrapasar a éste. Sola y primeramente en cuanto que el saber se in t e ri or i z a -y - r e c u e r d a a partir del ser inmediato encuentra por esta mediación la esencia." ${ }^{43}$ Cuando el saber se interioriza (Erinnerung) a partir del ser inmediato se encuentra la esencia, o sea, la esencia se muestra como tal, aparece, en la mediación que acontece en el mismo ser. La esencia ya está presente en lo inmediato, pero para verla hay que ver lo inmediato como "in-mediato". Es en la medida misma del ser y, en especial, en la línea nodal de relaciones de medida donde ya podemos ver la impronta misma de la esencia en la mediación de lo inmediato del ser. De esta manera, nos damos cuenta la imbricación entre ambos es total: ser y esencia, esencia y ser, son dos caras de una misma moneda (la moneda es el concepto). Además es interesante hacer notar que cuando la esencia se muestra, esto acontece porque ella misma se hace presente, o sea, "es", "inmediata"; lo sido en cuanto "es" se muestra para el análisis, pues de la otra forma siempre está como

\footnotetext{
${ }^{41}$ Hegel, G.W.F., Ciencia de la lógica, op. cit., p. 442.

${ }^{42}$ Badiou muestra el alcance de lo que Hegel entiende por Historia de modo radical, una historia que es política: "Pour Hegel en effet, l'exposition historique des politiques n'est pas une subjectivation imaginaire, c'est le réel en personne. Car l'axiome crucial de la dialectique telle qu'il la concoit est que 'le Vrai est le devenir de lui-même', ou, ce qui revient au même, 'le Temps est l'étre -lá du Concept'”. Badiou, A., "L'Idée du communisme”, en Badiou, A. y Žižek, S., L'Idée du Communisme, Paris, Lignes, 2010, p. 13.

${ }^{43}$ Hegel, G.W.F., Ciencia de la lógica, op. cit., p. 437.
} 
por "detrás" del aparecer inmediato del ser. Cuando el análisis mira a la esencia, el ser se vuelve "sido" y desaparece por "detrás" de la esencia porque ella es ahora inmediata para el análisis. Lo que está claro es que la mediación, la interiorización, es lo que nos permite ver lo que queremos ver y, a la vez, si nos damos cuenta siempre estamos en la interiorización, esto es, en la mediación metódica de lo inmediato. Y es ahí donde nos subjetivamos a diario y por siglos. Es la historia con todo lo que ella mienta y toda la negatividad que lleva en sí misma para avanzar afirmativamente. El crimen y el demonio con toda la barbarie va siendo parte del pastiche de lo Lógico y ello es lo que opera como potencia esencial que produce ortogonalmente la regla del ser, del presente: “... la trabazón infectante que nos fortalece para asumirnos sobre esta tierra" ${ }^{\prime 4}$. La esencia es el pasado y opera articulada con el ser que es el presente, son como dos planos en el carácter ortogonal que se relacionan ente sí: el presente del ser está tocado por el pasado esencial; lo inmediato a la luz de lo mediato, de las relaciones de medida que se han construido a lo largo de la historia y que luego se muestras como inmediatamente lo que son, como si siempre fueron así (es la raíz de todo dogma en el que se incardina la práctica del fanático). Esta regla que norma y constituye la realidad en su apariencia inmediata es una regla de suyo histórica que se ha realizado, se ha construido a lo largo del tiempo y con mucho dolor, ensayo, contingencia y error. El pasado de la esencia en su esenciar lleva dentro de sí todo el dinamismo potenciador de la negatividad. Si algo aparece como una punta de iceberg que es la regla que nos permite ser, movernos y crear, es porque esa misma regla esconde al iceberg entero con toda su pesadez dinámica que nunca se agota y que siempre está saliendo fuera de sí. Y la articulación entre estos dos momentos, punta de iceberg e iceberg, ser y esencia, es el concepto.

Hegel es muy claro en "La doctrina del concepto" de WdL: "Der Begriff ist von dieser Seite zunächst überhaupt als das Dritte zum Seyn und Wesen, zum Unmittelbaren und zu Reflexion anzusehen. Seyn und Wesen sind insofern die Momente seines Werdens; er aber ist ihre Grundlage und Warheit, als die Identität, in welcher sie untergegangen und enthalten sind" ${ }^{45}$. El concepto es la realización efectiva de la realidad en su desarrollo complejo (por eso, decíamos que era la polis para los mismos griegos), es, si se quiere, el futuro que va aconteciendo afirmativamente entre este reconocimiento entre presente (ser) y pasado (esencia), en punta de iceberg (inmediato, identidad) e iceberg mismo (mediación, reflexión). Como dice Ripalda

\footnotetext{
${ }^{44}$ Espinoza, R., "Epílogo: Políticamente habita el hombre”, en VVAA, Politicamente habita el hombre, Concón, Midas, 2009, p. 150.

${ }^{45}$ Hegel, G.W.F., Wissenschaft der Logik. Zweiter Band. Die Subjektive Logik (1816), op. cit., p. 11. "De este lado, hay que ver por lo pronto al concepto, en suma, como lo tercero respecto al ser y a la esencia, a lo inmediato y a la reflexión. En esa medida, ser y esencia son los momentos de su devenir, mientras que él es basamento y verdad de aquéllos, al ser la identidad en la que han caído, y en la que están contenidos". Hegel, G.W.F., Ciencia de la lógica. Volumen II: La lógica subjetiva. 3. La doctrina del concepto (1816), op. cit., p. 125.
} 
en su libro La nación dividida: “"El puro concepto', 'el abismo de la nada en que se hunde todo ser'- a que apunta como su culminación la conciencia burguesa y su filosofía reflexiva- supera ya tanto las abstracciones, a la vez vacías y excesivamente concretas, de la sociedad de consumo, como la dolorosa intimidad del absurdo. A él le corresponde reconstruir en la forma de la filosofía - superior no sólo a la inmediatez religiosa e histórica, sino también a las filosofías identificadas inconscientemente con la realidad de su tiempo y, por tanto, "dogmáticas" "46. La inmediatez propia del ser es propiamente tal a la luz de las mediaciones de la esencia que la permiten como tal. Y todo este proceso en afirmación es a la vez el concepto. Hegel lo dice así en "La doctrina del concepto": "Das abstract-Unmittelbare ist wohl ein Erstes; als diss Abstracte, ist es aber vielmeh ein Vermitteltes, von dem also, wenn es in seiner Wahrheit gefasst werden soll, siene Grundlage erst zu suchen ist. Diese muss daher zwar ein Unmittelbares seyn, aber so dass es aus der Aufhebung der Vermittlung sich zum Unmittelbaren genacht hat" ${ }^{\prime 7}$. La capa de presente que nos norma y regla y es la ciudad por antonomasia en la que somos (nuestro panóptico en el que vivimos y somos con todas nuestras creencias y representaciones) es a la vez desde esa no-ciudad, ese afuera contingente, que permite históricamente, con toda su resistencia, verdad e incertidumbre, ser. Y en ese dinamismo que no se resuelve, el concepto va generando la realidad misma, nacen las ideas; el diseño se realiza efectivamente, la innovación va diseñando modelos más lógicos, más humanos, más libres que van limpiando de opacidad, oscuridad y barbarie todas las zonas de la realidad (de lo racional y de lo real). Y en esa misma luz se dan zonas de más luz y de menos luz; son zonas campales de luminosidad pero con bordes, contracciones, curvaturas.

\section{Conclusión}

El método de lo Lógico busca radicalmente no solamente expresar lo inmediato del ser y reflexionarlo desde la historización de la esencia (esto sería la primera negación para Hegel), sino radicalmente, lo Lógico busca afirmar, diríamos, más fuertemente, producir, diseñar, crear lo real, esto es, el concepto (la segunda negación): "Es bestimmt sich frei; seine Verendlichung ist kein Übergehen, das nur in der Sphäre des Seyns Statt hat; es ist schöpferische Macht, als die absolute Negativität, die sich auf sich selbst bezieht... Somit ist es ein Setzen der Unterschiede selbst als

\footnotetext{
${ }^{46}$ Ripalda, J.M., La nación dividida. Raices de un pensador burgués: G.W.F. Hegel, Madrid, FCE, 1978, p. 250.

${ }^{47}$ Hegel, G.W.F., Wissenschaft der Logik. Zweiter Band. Die Subjektive Logik (1816), op. cit., p. 11. "Lo abstractamente inmediato es ciertamente algo primero; pero, al ser abstracto, es más bien algo mediado del que por ende, si debe ser captado en su verdad, hay que buscar primero su basamento. Por consiguiente, éste tiene que ser ciertamente algo inmediato, mas de modo tal que se haya hecho inmediato partiendo de la asunción de la mediación". Hegel, G.W.F., Ciencia de la lógica. Volumen II: La lógica subjetiva. 3. La doctrina del concepto (1816), op. cit., p. 125.
} 
allgemeiner, sich auf sich beziehender. Hierdurch werden sie fixirte, isolirte Unterschiede. Das isolirte Bestehen des Endlichen, das sich früher als sein Fürsichseyn, auch als Dingheit, als Substanz bestimmte, ist in seiner Wahrheit die Allgemeinheit, mit welcher Form der unendliche Begriff seine Unterschiede bekleidet,--eine Form, die eben einer seiner Unterschiede selbst ist. Hierin besteht das Schaffen des Begri$\mathrm{ffs}$, das nur in diesem Innersten desselben selbst zu begreifen ist" ${ }^{\text {"48 }}$. El concepto es creador y esto es fundamental para entender a Hegel desde muy joven y su pasado cercano a la Revolución francesa a su posterior trabajo filosófico; trabajo muy serio, de alta precisión, es como un gran pulidor de conceptos, en especial, del concepto de "concepto" (Begriff); lo pule por años y lo pule para que muestre toda su potencia creadora $^{49}$. El concepto es creador y esto no es metáfora alguna, sino lo propio de él y crea a la luz de la inmediatez del ser y de la reflexividad de la esencia. En esa inmediatez mediatizada acontece el concepto en su carácter concreto creador.

La afirmación conceptual de lo Lógico surge de la negación de la negación, como le gusta decir a Hegel, pero afirmación radical al final de cuentas. El ser es negado en su in-mediatez por la mediación misma. Y en ello se da la negación afirmadora de esa negación anterior. Es, si queremos, la propia esencia afirmada; es afirmar la segunda negación. Es el gran Sí de Hegel. Más profundo que el dolor, parodiando a Nietzsche, está la propia afirmación de esa negación, por eso Hegel es el pensador de lo concreto y lleva dentro de sí la contingencia y lo que no se puede sistematizar de ésta dentro de lo Lógico. Así lo dice en "La doctrina del concepto" de la WdL: "Der Begriff zeigt sich obenhin betractet, als die Einheit des Seyns und Wesens. Das Wesen ist die erste Negation des Seyns, das dadurch zum Schein geworden ist, der Begriff ist die zweyte, oder die Negation dieser Negation; also das wiederhergestellte Seyn, aber als die unendliche Vermittlung und Negativität desselben in

\footnotetext{
${ }^{48}$ Hegel, G.W.F., Wissenschaft der Logik. Zweiter Band. Die Subjektive Logik (1816), op. cit., pp. 36-37. "Él se determina [y destina] libremente; su conversión en algo finito no es ningún transitar, cosa que sólo en la esfera del ser tiene lugar; él es potencia creadora, entendida como la negatividad absoluta que se refiere a sí misma... Con esto, él es un acto de poner las diferencias mismas como diferencias universales, como diferencias que se refieren a sí. De este modo, vienen a ser diferencias fijadas, aisladas. El aislado consistir de lo finito, que anteriormente se determinó como su ser-para-sí, y también como coseidad, como sustancia, es en su verdad la universalidad, forma con la cual reviste el concepto infinito sus diferencias: una forma que es ella misma, justamente una de sus diferencias. En esto consiste el crear del concepto, un crear que ha de ser concebido solamente dentro de esta más íntima entraña del concepto mismo". Hegel, G.W.F., Ciencia de la lógica. Volumen II: La lógica subjetiva. 3. La doctrina del concepto (1816), op. cit., p. 156.

49 “'Hasta 1800 Hegel no quiso ser filósofo, sino revolucionario... Pero de la 'Revolución' - que coge en la universidad- a las guerras napoleónicas la historia se precipita; entre 1799 y 1801 Hegel pierde pie. Entonces, con 30 años, se vuelve a la filosofía para que le enseñe a vivir en una etapa casi febril de publicación”. Ripalda, J.M., "Contraportada”, en Hegel, G.W.F., El joven Hegel. Ensayos y esbozos, Madrid, FCE, 2014.
} 
sich selbst" ${ }^{\prime 50}$. El concepto, esto es, lo Lógico afirma radicalmente la realidad en su negatividad y, en esto, su carácter universal mienta una universalidad concreta que se afirma y es creadora: "Leben, Ich, Geist, absoluter Begriff, sind nicht Allgemeine nur als höhere Gattungen, sondern Konkrete" ${ }^{51}$.

El sistema de pensamiento de Hegel es totalmente importante hoy y todavía tiene mucho que dar de sí ya para la filosofía como para las ciencias y la teoría política, pues en Hegel se permite ver cómo la historia opera en el plano inmanente de la mera presencia del presente del ser, punta del iceberg en el que vivimos, como ideología, sistema de referencias, prácticas naturalizadas, dogmas, etc. Siempre se puede esperar en Hegel un cambio en esa punta de iceberg que nos regla y nos mide, que es el ser en su aparecer y brillar, ésta siempre se puede modificar: el pasado, la esencia, no está al fondo escrito para siempre de una forma determinada, sino que siempre está dando vida; el iceberg de la esencia con todo su negatividad abre y vuelve a abrir la regla del ser, luego ella nunca será del todo rígida, la ideología nunca nos gobierna en forma absoluta. Así la filosofía de Hegel, desde esta comprensión de lo Lógico, puede entenderse desde una dimensión revolucionaria a la altura de los tiempos, de una posible revolución que devela todo rasgo ideológico. Lo Lógico, el elemento de lo Lógico, en su expresión compleja que hemos explicado, siempre dará de sí un nuevo momento con mayor información que nos permitirá tomar decisión para diseñar el futuro. ${ }^{52}$ Pues solamente en tanto acontece lo Lógico como concepto nos movemos en elemento de lo libre y de ahí la toma de decisiones y el diseño de lo real. Hegel lo dice en formula sintética: "Das reine Begriff ist das absolut unendliche, unbedingte und freye" ${ }^{\prime \prime 3}$.

\footnotetext{
${ }^{50}$ Hegel, G.W.F., Wissenschaft der Logik. Zweiter Band. Die Subjektive Logik (1816), op. cit., p. 29. "El concepto se muestra, según lo considerado anteriormente, como la unidad del ser y de la esencia. La esencia es la primera negación del ser, que ha venido a ser por ello apariencia; el concepto es la segunda, o sea la negación de esa negación; por tanto, el ser restablecido, pero como la infinita mediación y negatividad del mismo en sí mismo". Hegel, G.W.F., Ciencia de la lógica. Volumen II: La lógica subjetiva. 3. La doctrina del concepto (1816), op. cit., p. 145.

${ }^{51}$ Ibíd., p. 36. "Vida, Yo, espíritu, concepto absoluto, no son universales solamente como géneros superiores, sino que son concretos".

${ }^{52}$ Tal como ya decía Hegel al comienzo de FEN, en el Prólogo: “... por existir la filosofía, esencialmente, en el elemento de lo universal, que lleva dentro de sí lo particular, suscita más que otra ciencia cualquiera la apariencia de que en el fin o en los resultados últimos se expresa la cosa misma, e incluso se expresa en su esencia perfecta, frente a lo cual el desarrollo parece representar, propiamente, lo no esencial." Hegel, G.W.F., Fenomenología del espíritu, op. cit., p. 7.

${ }^{53}$ Hegel, G.W.F., Wissenschaft der Logik. Zweiter Band. Die Subjektive Logik (1816), op. cit., p. 33. "El concepto puro es lo absolutamente infinito, incondicionado y libre". Hegel, G.W.F., Ciencia de la lógica. Volumen II: La lógica subjetiva. 3. La doctrina del concepto (1816), op. cit., p. 151.
} 


\section{Referencias bibliográficas}

BADIOU, A., y Žižek, S. L'Idée du Communisme, Lignes, Paris, 2010.

CASTELLS, M. "Creatividad, innovación y cultura digital. Un mapa de sus interacciones", en Telos, cuadernos de comunicación e innovación, 77, 2008, pp. $50-52$.

CASTELLS, M. "La apropiación de las tecnologías: cultura juvenil en la era digital", en Telos, cuadernos de comunicación e innovación, 81, 2009, pp. 111-113.

CASTELLS, M. "La democràcia en l'era d'Internet", en Via valors, Idees, Actituds: Revista del Centre d'estudis Jordi Pujol, 12, 2010, pp. 7-13.

DELEUZE, G. Spinoza y el problema de la expresión, Atajos, Barcelona, 1999.

DUQUE, F. Historia de la Filosofia Moderna. La era de la crítica, Akal, Madrid, 1998.

DUQUE, F. (editor) Hegel: La Odisea del Espíritu (1807-2007), Círculo de Bellas Artes, Madrid, 2010.

ESPINOZA, R. "Una lectura 'desmesurada' de la Ciencia de la lógica de Hegel: en torno a "la línea nodal de relaciones de medida", Philosophica, 30, 2007, pp. 89-102.

ESPINOZA, R. "Arte y religión en la Phänomenologie des Geistes de Hegel a la luz de la Wissenschaft der Logik...", Daimon, 43, 2008, pp. 71-91.

ESPINOZA, R. "Epílogo: Políticamente habita el hombre", en VVAA, Políticamente habita el hombre, Midas, Concón, 2009.

ESPINOZA, R. Hegel y las nuevas lógicas del mundo y del Estado. ¿Cómo se es revolucionario hoy?, Akal, Madrid, 2016.

FINK, E., Hegel: Interpretaciones fenomenológicas de la Fenomenología del espiri$t u$, Herder, Barcelona, 2011.

FUKUYAMA, F. El fin de la historia y el último hombre, Davinci, Barcelona, 2010.

JAMESON, F. Reflexiones sobre la postmodernidad, Abada, Madrid, 2010.

HEGEL, G. W. F. Fenomenología del espiritu, FCE, México, 1966.

HEGEL, G.W.F. Wissenschaft der Logik. Ersted Band. Die objetive Logik (1812/1813). Herausgegeben von Friedrich Hogemann und Walter Jaeschke, Felix Meiner, Hamburg, 1978.

HEGEL, G.W.F. Wissenschaft der Logik. Zweiter Band. Die Subjektive Logik (1816), Herausgegeben von Friedrich Hogemann und Walter Jaeschke, Felix Meiner Verlag, Hamburg, 1981.

HEGEL, G.W.F. Wissenschaft der Logik. Erster Teil. Die Objetive Logik. Ersted Band. Die Lehre vom Sein (1832). Herausgegeben von Friedrich Hogemann und Walter Jaeschke, Felix Meiner, Hamburg, 1985.

HEGEL, G.W.F. Rasgos Fundamentales de la Filosofía del Derecho, Biblioteca Nueva, Madrid, 2000. 
HEGEL, G. F. W. Enciclopedia de las ciencias filosóficas, Alianza, Madrid, 2008.

HEGEL, G.W.F. Ciencia de la lógica. Volumen I: La lógica objetiva (1812/1813), Abada, Madrid, 2011.

HEGEL, G.W.F. Ciencia de la lógica. Volumen II: La lógica subjetiva. 3. La doctrina del concepto (1816), Abada, Madrid, 2015.

HEIDEGGER, M. Sein und Zeit, Vittorio Klostermann, Franckfurt am Main, 1977.

HEIDEGGER, M. Identidad y diferencia, Anthropos, Barcelona, 1988.

HEIDEGGER, M. Seminario de Le Thor (1969), Alción Editora, Córdoba, 1995.

HEIDEGGER, M. Hitos, Alianza, Madrid, 2001.

HEIDEGGER, M. Hegel, Prometeo, Buenos Aires, 2005.

HEIDEGGER, M. Caminos del bosque, Alianza, Madrid, 2010.

PINKARD, T. Hegel's Naturalism. Mind, Nature, and the Final Ends of Life, Oxford University Press, New York, 2012.

PIPPIN, R. Hegel On Self-Consciousness. Dessire and Death in the Phenomenology of Spirit, Princeton University Press, New Jersey, 2011.

PIPPIN, R. Hegel's Practical Philosophy. Rational Agency as Ethical Life, Cambridge University Press, Cambridge, 2010.

RIPALDA, J.M. La nación dividida. Raíces de un pensador burgués: G.W.F. Hegel, FCE, Madrid, 1978.

RIPALDA, J.M. "Introducción”, en HEGEL, G.W.F., Filosofia Real, FCE, Madrid, 2006.

RIPALDA, J.M. “Contraportada”, en HEGEL, G.W.F., El joven Hegel. Ensayos y esbozos, FCE, Madrid, 2014.

TOSCANO, A. Fanaticism. On the Uses of an Idea, Verso, London, 2010.

VALLS PLANA, R. Del yo al nosotros. Lectura de la Fenomenología del Espiritu de Hegel, Editorial Estela, Barcelona, 1971.

VITIELLO, V. "Por qué Hegel, Hoy", en ESPINOZA, R. (ed.), Hegel. La transformación de los espacios sociales, Midas, Concón, 2012.

ŽIŽEK, S. El más sublime de los histéricos, Paidós, Barcelona, 2013

ZUBIRI, X. Inteligencia sentiente, Alianza, Madrid, 1980.

ZUBIRI, X. Inteligencia y Logos, Alianza, Madrid, 1982.

ZUBIRI, X. Inteligencia y Razón, Alianza, Madrid, 1983.

ZUBIRI, X. Los problemas fundamentales de Occidente, Alianza, Madrid, 1994.

ZUBIRI, X. Naturaleza, Historia, Dios, Alianza, Madrid, 2007.

ZUBIRI, X. Sobre la esencia, Alianza, Madrid, 2008. 